\title{
Papillomavirus-associated balanoposthitis
}

\author{
A Wikström, G von Krogh, M-A Hedblad, S Syrjänen
}

\begin{abstract}
Objective-To assess whether there might be an association between genital papillomavirus infection (GPVI) and balanoposthitis.

Design-Retrospective HPV DNA examination of biopsy specimens from 23 men suffering from balanoposthitis and exhibiting acetowhite lesions that were penoscopically and histologically concurrent with HPV infection.
\end{abstract}

Setting-The STD clinics at Karolinska Hospital and South Hospital, Stockholm, Sweden.

Participitants-Randomly selected men attending with long-lasting and/or recurrent penile symptoms and exhibiting a clinical picture of balanoposthitis, who revealed a penoscopical and histopathological picture of epidermal lesions that were concordant with accepted criteria for typical or conspicuous GPVI. Asymptomatic controls were selected retrospectively on the basis of identical penoscopy and histology criteria.

Results-A history of previous condylomata was obtained in eight (35\%) of 23 men. At penoscopic evaluation tiny condylomatous lesions were observed in five $(22 \%)$ patients. The in situ hybridisation (ISH) assay using specific probes for the HPV types $6 / 11,16 / 18,31 / 33$ and 42 was positive in $13 / 23(56 \%)$ of the patient samples, but in only $26 \%$ of the 19 control samples. In patient biopsies the oncogenic HPV types $16 / 18$ and/or $31 / 33$ were found in 7/13 samples, whereas HPV 6/11 and/or 42 were present in another six cases. PCR performed on the ten ISH negative patient biopsies, were negative in all cases.

Conclusion-Symptoms included redness, itching, burning, tenderness, dyspareunia, fissuring and in two cases penile oedema and inguinal adenopathy. All patients fulfilled penoscopical and histopathological criteria for HPV infection. We demonstrate some tentative evidence that HPV might be associated with long-lasting balanoposthitis, although our data still are circumstantial for a causative association. The results also elucidate the diversity in clinical presentation of GPVI.

(Genitourin Med 1994;70:175-181)

Introduction

Altogether $28 \mathrm{HPV}$ genotypes exhibit a pref- erential trophism for the genitoanal area (genitoanal papillomavirus infection; GPVI) ${ }^{1}$ The typical condylomata acuminata (genital warts), known since ancient times, ${ }^{2}$ appear to be predominantly caused by the HPV types 6 and 11 , which are at present classified as "low risk" HPV types owing to their lack of significant oncogenic potential. ${ }^{3}$ However, these warts merely represent the "tip of the iceberg"4 5; GPVI most often gives rise to lesions that are undetectable by naked eye examination unless visualised by application of an aqueous solution of $3-5 \%$ acetic acid. ${ }^{6-8}$

While some acetowhite lesions are associated with HPV 6 and 11 or with other "low risk" types such as HPV 42, others are induced by potentially oncogenic "high risk" types such as HPV 16, 18, 31 and $33 .{ }^{78}$ The relative proportion of low versus high risk HPV types in acetowhite lesions varies in different studies. ${ }^{67}$

Varying degrees of dysplastic epithelial transformation, referred to as intraepithelial neoplasia, commonly occur in the genital area through HPV influence. The cervix uteri represents a locus minoris for the development of persistent and progressive dysplasia (cervical intraepithelial neoplasia; CIN) with a relatively high potential for progression into invasive cancer when associated with oncogenic HPV types. ${ }^{910}$

Most research on males afflicted with acetowhite penile lesions has focused on the potential role of the males representing vectors for spread of the infection to the female population, ${ }^{711-18}$ while less attention has been given to the potential morbidity for the male counterpart. Although acetowhite penile lesions apparently mostly are asymptomatic, they may in some cases give rise to symptoms such as itching, burning and dyspareunia. ${ }^{6}$

Recently, diagnostic criteria for typical and conspicuous acetowhite penile lesions, as evaluated by colposcopic magnification ("penoscopy"), have been discussed in detail. Yet histopathology evaluation appears to represent the "gold standard" for a final verification of underlying GPVI in an acetowhite lesion. Benign epidermal hyperplasia and various degrees of intraepithelial neoplasia are parts of a continuous morphological spectrum. ${ }^{1920}$ A pathognomonic histology entails the presence of an acanthotic epidermis with the occurrence of koilocytosis in the stratum spinosum. Additional conspicuous criteria entail parakeratosis and dilated dermal capillaries. The existence of intraepithelial neoplasia is common, and may or may not be associated with koilocytosis. Intraepithelial neoplasia on the outer genitals in young adults is almost
Address correspondence to: Dr A Wikström

Accepted for publication 16 January 1994 
Table 1 Symptoms and signs in 23 men exhibiting penoscopic findings indicating HPV infection

\begin{tabular}{lrlr}
\hline Symptoms & \multicolumn{3}{l}{ Signs } \\
\hline burning & $12(52 \%)$ & erythema & $21(91 \%)$ \\
fissuring & $10(43 \%)$ & fissuring & $8(35 \%)$ \\
redness & $9(39 \%)$ & oedema & $1(4 \%)$ \\
discomfort during & $9(39 \%)$ & inguinal adenopathy & $1(4 \%)$ \\
intercourse & & & \\
tenderness & $6(26 \%)$ & & \\
itching & $5(22 \%)$ & & \\
penile swelling & $1(4 \%)$ & & \\
inguinal pain & $1(4 \%)$ & & \\
\hline
\end{tabular}

unequivocally associated with HPV infection; while low risk HPV types occasionally are associated with mild to moderate dysplastic changes, severe dysplasia strongly indicates the presence of high risk HPV types. ${ }^{20-22}$

Available HPV DNA hybridisation assays are of significant investigative relevance for type-specific HPV detection but differ regarding sensitivity, specificity and applicability. ${ }^{23}$ The particular benefit of the in situ hybridisation (ISH) technique is that a correlation can
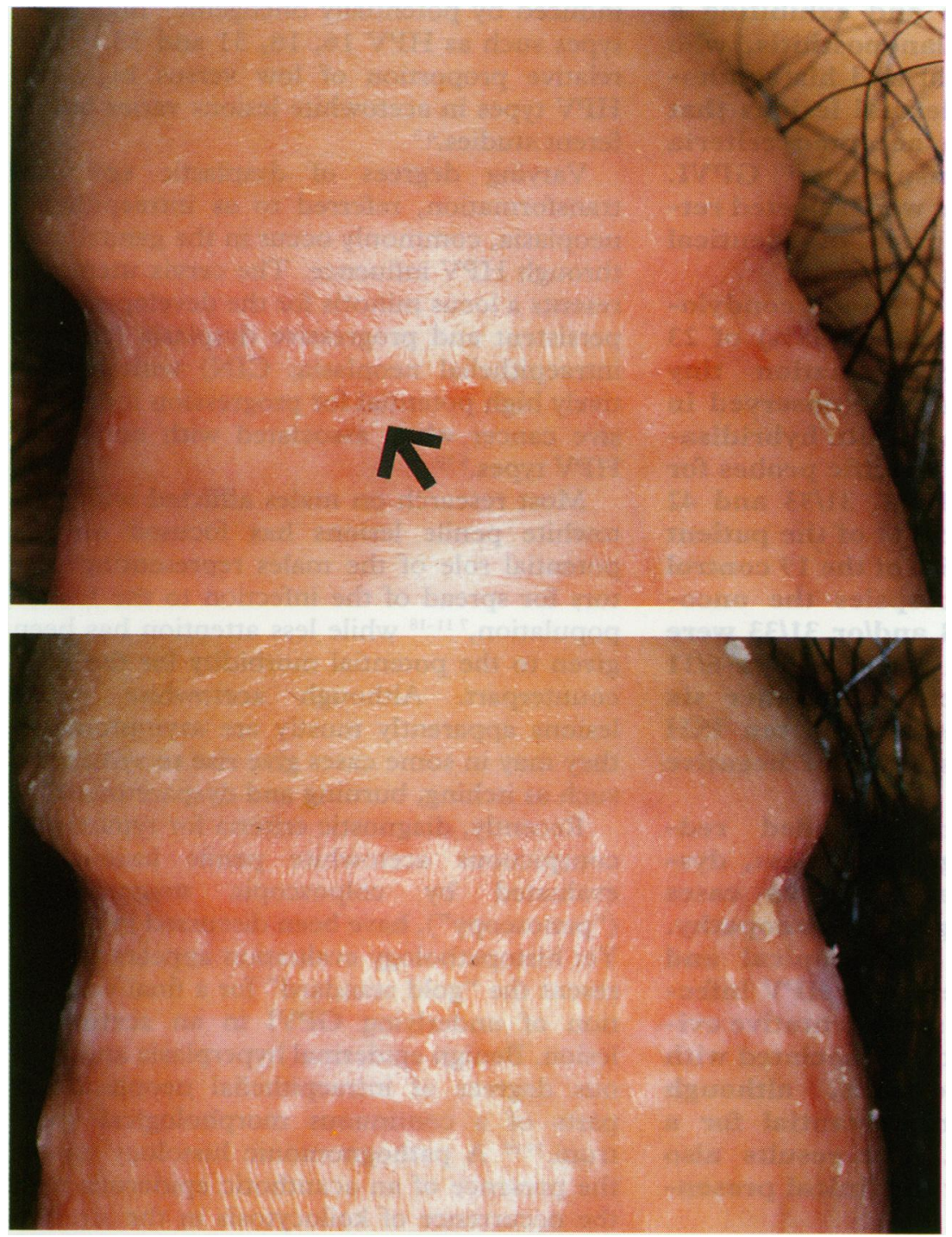

Figure 121 year old male with no history of previous STD. During a 4-months period noted erythematous patches in the sulcus coronarius and periodically experienced some dyspareunia associated with tenderness of the afflicted area. Penoscopy revealed maculopapulous erythematous lesions some of which exhibiting typical punctuated capillaries ( $a$; arrow), being sharply demarcated using the acetic acid. The biopsy showed a picture of PIN II (b), but no HPV DNA was detected in the ISH assay. Diathermy performed at three occasions during a 3-months period, thereafter free from symptoms during a 6 months follow-up period. be made between presence of HPV DNA and underlying histopathological morphology. ${ }^{23}$ However, although highly specific, the sensitivity of ISH is relatively low, allowing a detection of $>10$ virions per cell. ${ }^{24}$ The polymerase chain reaction (PCR) is the most sensitive assay, as the amplification allows one $\mathbb{\Phi}$ specific viral genome to be detected among 100.000 cells. ${ }^{25}$ However, sensitivity may be influenced by the type(s) of primer(s) and technical handling. ${ }^{26}$

In the present study we have analysed retrospectively biopsy specimens taken from $\overrightarrow{\bar{B}}$ either penoscopically typical or conspicuous 0 acetowhite lesions of 23 men attending due to 흠 long-lasting and/or recurrent penile symp- $\frac{\bar{s}}{\vec{D}}$ toms. All samples were investigated using $\stackrel{\mathbb{\Omega}}{\Omega}$ conventional light microscopy and in situ i hybridisation; ISH negative cases were further $\vec{\circ}$ analysed with HPV consensus PCR primers. Specimens collected from asymptomatic men $\vec{\omega}$ afflicted with penile acetowhite lesions of exhibiting equivalent penoscopic signs, were considered as controls and were investigated $\stackrel{\circ}{\omega}$ with ISH.

\section{Materials and methods}

Patients The study includes 23 randomly

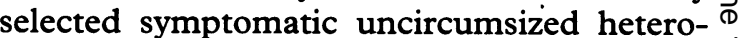
sexual men examined at the STD clinics at $\vec{\emptyset}$ the Karolinska Hospital and at the South Hospital in Stockholm, Sweden, during a four-year period (1987-1991); all of the men suffered from long-lasting and/or recurrent penile itching, burning, tenderness, dyspareunia, oedema and/or inguinal pain (table 1). Clinical signs entailed varying degrees of $\overrightarrow{\overrightarrow{0}}$ macroscopically apparent balanoposthitis including erythema, and in some cases the occurrence of fissures, oedema and/or inguinal adenopathy as well. Urethral speci- $\stackrel{\text { ? }}{=}$ mens were collected for direct microscopy (methylene blue staining) at high-power view ( $\times 1000)$, and for cultures of Neisseria gonorrhoeae and for ELISA assay detection of $\mathrm{O}$ Chlamydia trachomatis (Syva Scandinavia, Stockholm, Sweden). Concurrent syphilis was ruled out by the standard VDRL test, and HIV infection by ELISA technique (Abbott Scandinavia, Stockholm, Sweden). According to current clinical routine in our setting, all $\mathrm{\omega}$ men exhibiting signs of balanoposthitis were prescribed topical anti-inflammatory therapy

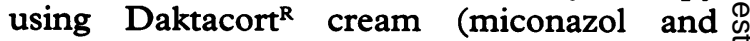
hydrocortison; Janssen Pharmaceutica, Belgium) twice daily for two weeks. The present study only includes men who followed $\stackrel{\odot}{\circ}$ these instructions and who subsequently $\mathbb{D}$ promptly returned, and in whom persistent acetowhite lesions were detected at reevalua- 8 tion at this time. Persistent acetowhite lesions were classified penoscopically as being either typical or conspicuous of GPVI. Lesions were classified as typical when they exhibited a well demarcated, slightly elevated border as well as discernible punctuated capillaries, while lesions were categorised as conspicuous if they displayed identical criteria except for the presence of punctuated capillaries. ${ }^{6}$ 
Table 2 Result of the in situ hybridisation (ISH) assay with type-specific HPV probes in 23 biopsies

\begin{tabular}{lc}
\hline HPV 6/11 & $2(9 \%)$ \\
HPV 16/18 & $4(17 \%)$ \\
HPV 31/33 & $3(13 \%)$ \\
HPV 42 & $3(13 \%)$ \\
HPV 6/11+42 & $1(4 \%)$ \\
Total & $13(56 \%)$ \\
\hline
\end{tabular}

Controls As controls, 19 asymptomatic men were retrospectively selected among biopsy samples previously collected from 91 men presenting with penoscopically typical or conspicuous acetowhite penile lesions ${ }^{6}$ exhibiting histopathological signs typical for GPVI, that is, the presence of koilocytosis and/or PIN. These samples were analysed with ISH. The presence of concurrent STDs and urethritis was tested analogously to that of the patients.

Biopsies One biopsy specimen was taken from each patient after local anaesthesia with lidocaine- $\mathrm{HCl} 5 \mathrm{mg} / \mathrm{ml}$ (Astra, Sweden). In the patient group biopsies were generally somewhat larger $(4-6 \mathrm{~mm})$ compared with those of the controls $(2-3 \mathrm{~mm})$.

Light microscopic evaluation The specimens were fixed in neutral $10 \%$ formalin and paraffin embedded for routine histology. All were examined by the same dermatohistopathologist (M.H.). Specimens showing epidermal hyperplasia, acanthosis, parakeratosis and koilocytosis were classified as typical, whereas those exhibiting all criteria except for koilocytosis were classified as histopathologically conspicuous, unless they displayed any sign of intraepithelial neoplasia (PIN), when they were also classified as typical. Any PIN was further graded as degree I, II or III, based on accepted criteria entailing the extent of dysplastic involvement of the epithelium..$^{72}$ Any dermal inflammatory infiltrate was graded as being mild, moderate or severe. Fifteen biopsies were also PAS stained for signs of potentially persistent fungal infection.

Hybridisation assays Paraffin-embedded biopsy specimens from all of the patients were examined with ISH, and in ISH negative samples also with PCR; ISH was performed on the control biopsies.

ISH In situ hybridisation was performed to detect HPV DNA specific for the HPV types $6 / 11,16 / 18,31 / 33$ and 42 using a biotinylated whole genomic probe as earlier described. ${ }^{24} 28$ Shortly, after deparaffinisation and deproteinisation, the sections were simultaneously denatured with the hybridisation mixture containing $2 \mu \mathrm{g} / \mathrm{ml}$ biotinylated probe in $2 \times \mathrm{SSC}$, $50 \%$ formamide, $0,4 \mathrm{mg} / \mathrm{ml}$ herring sperm DNA, $10 \%$ dextran sulphate. After overnight hybridisation at $55^{\circ} \mathrm{C}$, the sections were subsequently washed with $2 \times S S C$ at room temperature, $0.2 \times \mathrm{SSC}$ at $60^{\circ} \mathrm{C}$ and $2 \times \mathrm{SSC}$ at room temperature. The hybrids were detected by streptavidin-alkaline phosphatase (Amersham International plc, Amersham, UK) complex using NBT and BCIP as chromagen.

$P C R$ One to several $5-\mu \mathrm{m}$ thick sections were cut to achieve an average surface area of 1 $\mathrm{cm}^{2}$ Before slicing, the excess paraffin was cut off from the block. The sections were directly placed into a 500- $\mu 1$ microfuge tube. The paraffin was extracted twice with $500 \mu$ l xylene by mixing gently for 5 minutes at room temperature, followed by washes with absolute ethanol to remove xylene. After removing the ethanol, a few drops of acetone was added to each tube to be evaporated. On to dried samples, $50 \mu 1$ of sterile distilled water was added. The pellet was gently resuspended and boiled for ten minutes. The tubes were transferred on ice and centrifuged to pellet the undissolved material. Samples were used immediately or stored at $-20^{\circ} \mathrm{C}$ until used. For PCR, $15 \mu \mathrm{l}$ of supernatant was used. PCR was done in $50 \mu \mathrm{l}$ of the reaction mixture $(50 \mathrm{mM} \mathrm{KCl}, 10 \mathrm{mM}$ TrisHCl $(\mathrm{pH}$ 8.8), $1.5 \mathrm{mM} \mathrm{MgCl}_{2}, 0,1 \%$ Triton X-100), 20 pmol of primers, $200 \mathrm{mU}$ of each deoxynucleotide triphosphate and 0.75 units of DynaZyme DNA polymerase (Finnzymes, Espo, Finland).

For the detection of HPV DNA the consensus primers from the $\mathrm{L} 1$ region were used as described by Manos et al in 1989. ${ }^{29}$ The specificity of the amplified PCR product was confirmed by digestion with the restriction enzymes Pst 1 and Rsa I (BioLabs).

\section{Results}

Patients The mean age of the men was 29 (range 19-58) years; median age was 26 years. No case of urethritis was detected, and all men had negative tests for Neisseria gonorrhoeae, Chlamydia trachomatis, syphilis and HIV. A history of previous condylomata was reported by eight (35\%) of the men.

Penoscopically typical GPVI lesions were detected in 10 (43\%), and conspicuous changes in another $13(57 \%)$ of the 23 men.

Penile symptoms included burning in 12 $(52 \%)$, redness in nine $(39 \%)$, some degree of discomfort during intercourse in nine $(39 \%)$, tenderness in six $(26 \%)$ and itching in five $(22 \%)$ of the men (table 1$)$. Ten $(43 \%)$ of the men complained about epithelial fissures that had periodically been painful to some degree and frequently given rise to periodic moderate dyspareunia. One man (4\%) had experienced a sudden onset of penile swelling that lasted for some days. Another patient reported recurrent inguinal pain for about a month. Exact information about the duration of symptoms was available in 18 patients; the mean was 11 and the median four (range 0-48) months.

Clinical investigation demonstrated a localised and partial erythema of the glans, the sulcus coronarius, the fraenulum and/or the foreskin in $21(91 \%)$ of the men, while eight men (35\%) exhibited a penoscopically apparent fissuring (table 1). One man (4\%) presented with a marked penile oedema (fig 2a). Another patient had been referred to the department because of unexplainable inguinal adenopathy that was confirmed by us at attendance. Penoscopy revealed that five of the men $(22 \%)$ were concurrently afflicted with tiny acuminate or papular lesions that were inapparent by naked eye examination. Only one of these five men had a history of previous condylomata.

Altogether 21 (91\%) of the 23 biopsy samples showed light microscopic typical features of HPV infection; koilocytosis was detected in $15(71 \%)$ of the typical samples. 
The histology was classified as conspicuous in another two cases (9\%). PIN was noted in 17 of the biopsies (74\%); PIN I occurred in six $(26 \%)$, PIN II in seven (30\%) and PIN III in four $(17 \%)$ samples. Of samples exhibiting PIN, 11/17 (65\%) exhibited focal areas of koilocytosis, occurring either in direct association with dysplasia, or occurring in adjacent areas of the samples. Various degrees of dermal lymphocytic inflammatory infiltrates were present in $17(74 \%)$ of the cases; mild, moderate and severe in seven (30\%), eight (35\%) and two (9\%) biopsies, respectively.

ISH was positive in $13(56 \%)$ of the 23 samples, two being positive for HPV 6/11, four for HPV 16/18, three for HPV 31/33 and three for HPV 42. Double infection with HPV $6 / 11$ and 42 was present in one case (table 2). Of samples that were histopathologically typical for HPV infection, 11/21 (52\%) were HPV positive. The ISH was also positive in both of


Figure 224 year old subjectively healthy male with no history of previous STD attended because his steady female partner was diagnosed with GPVI. No warts were found but he was treated with diathermy due to presence of minute penoscopically typical GPVI lesions in the sulcus coronarius. At a 2-months follow-up still subjectively healthy, the acetic acid test being negative and penoscopy evaluation indicating normal conditions. After another two months a sudden onset of oedema afflicting the foreskin and the distal part of the penile shaft (a). After retraction of the foreskin and performance of the acetic acid test $a$ conspicious acetowhite lesion appears (b; arrow). Genital examination otherwise normal. Treated orally with norfloxacin and with topical anti-inflammatory therapy. Within a week complete disappearance of oedema. Thereafter free from symptoms during a 2-years follow-up period. This male harboured HPV 42, and the biopsy exhibited PIN grade I. the two samples histologically classified as conspicuous. The PCR assay was performed on ten ISH negative biopsies, in all cases with a negative result. Candida was not detected in any of 15 PAS stained samples.

In table 3, penoscopy, histopathology and ISH findings among the 23 men included in the study are summarised. Penoscopically, a conspicuous picture was found in $41 \%$ and a typical appearance in $59 \%$ of the PIN cases.

Controls The mean age, 25 years (range $\bar{\Phi}$ 20-31 years), was somewhat lower than that of the patients; median age was 24 years. No $\overrightarrow{\vec{S}}$ cases of urethritis or other STDs were diag- $\overrightarrow{0}$ nosed among the controls. These men neither $\frac{\sigma}{\sigma}$ presented themselves with symptoms, nor had $\frac{\bar{\omega}}{5}$ a history of or clinical signs of balanoposthitis. $\stackrel{\mathbb{D}}{\Omega}$ However, a history of previous condylomata क was reported by $15(79 \%)$ of the men.

Penoscopically, $12(63 \%)$ of the men exhibited typical and seven (37\%) conspicu- $\vec{\omega}$ ous acetowhite lesions. Furthermore, by penoscopy four $(21 \%)$ of the men appeared to be concurrently afflicted with tiny acuminate or papular warts.

Histopathological examination showed typ- जे ical HPV signs in all of the controls; koilocy- 을 tosis was present in $15(79 \%)$ and some degree $\overrightarrow{ }$ of PIN in $12(63 \%)$ samples. PIN I, II and III

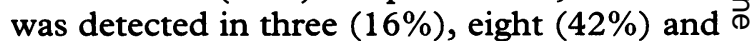
one $(5 \%)$ of the biopsies, respectively. Of $\vec{\theta}$ biopsies revealing any PIN, 8/12 (67\%) concurrently exhibited focal areas of koilocytosis. Dermal inflammatory infiltrates were present in $17(89 \%)$ samples, graded as mild, moderate and severe in eight $(42 \%)$, seven $(37 \%)$ and two (10\%) biopsies, respectively.

The ISH assay was positive in $5 / 19(26 \%) \overrightarrow{\overrightarrow{0}}$ cases, with HPV 16 present in one (5\%) and 3 HPV 42 in four $(21 \%)$ of the biopsy specimens.

\section{Discussion}

Although no classical condylomas had been observed by the patients at the time of investi- 0 gation, as much as $35 \%$ of the patients and $79 \%$ of the controls gave a positive history of previous condylomata therapy. Furthermore, tiny acuminate and/or papular warty lesions, o unnoticed by the patients, were detected by $N$ penoscopy in $22 \%$ and $21 \%$ of patients and $\mathrm{\omega}$ controls, respectively. As the present study is based on penoscopic criteria that previously have demonstrated a high predictive value for $\mathscr{\oplus}$ the detection of acetowhite GPVI lesions, ${ }^{6}$ and all 23 patient biopsies revealed one or more histological signs of HPV influence, we $\overrightarrow{\mathbb{D}}$ are inclined to believe that the present material $\mathbb{\Phi}$ represents true cases of biologically active HPV-associated lesions. This premise is further underscored by the fact that koilocytosis was present in 15 (65\%) and some degree of PIN in 17 (74\%) of the 23 patient biopsies. In this respect, corresponding figures among the controls were highly congruent, with koilocytosis and/or PIN detected in $79 \%$ and $63 \%$, respectively. Of the 17 patient biopsies exhibiting any degree of PIN, 11 (65\%) also exhibited concurrent koilocytosis; the corresponding figure for controls was 8 of 12 
Table 3 Summary of the results of penoscopy, histopathology and in situ hybridisation (ISH) for 23 men

\begin{tabular}{|c|c|c|c|c|c|c|c|c|c|c|c|c|}
\hline \multirow[b]{2}{*}{$\begin{array}{l}\text { Patient } \\
\text { number }\end{array}$} & \multirow[b]{2}{*}{$\begin{array}{l}\text { Age } \\
\text { (years) }\end{array}$} & \multirow{2}{*}{$\begin{array}{l}\text { Duration of } \\
\text { symptoms } \\
\text { (months) }\end{array}$} & \multirow[b]{2}{*}{$\begin{array}{l}\text { Previous } \\
\text { condylomas }\end{array}$} & \multicolumn{3}{|c|}{ Penoscopy } & \multicolumn{5}{|c|}{ Histopathology } & \multirow[b]{2}{*}{$I S H$} \\
\hline & & & & Typical & Conspicuous & $\begin{array}{l}\text { Concurrent } \\
\text { microcondylomas }\end{array}$ & Typical & Conspicuous & Koilocytosis & $P I N$ & $\begin{array}{l}\text { Dermal } \\
\text { inflammation }\end{array}$ & \\
\hline 1 & 21 & 4 & & $x$ & & & $x$ & & $x$ & II & - & - \\
\hline 2 & 33 & 4 & $x$ & $x$ & & & $x$ & & & I & + & - \\
\hline 3 & 24 & 6 & & & $x$ & $x$ & $x$ & & $x$ & I & - & $6 / 11+42$ \\
\hline 4 & 24 & 0 & $x$ & & $x$ & & $x$ & & $x$ & I & - & 42 \\
\hline 5 & 25 & 1 & & & $x$ & $x$ & $x$ & & $x$ & 0 & - & 42 \\
\hline 6 & 45 & 1 & & & $x$ & & & $x$ & & 0 & ++ & $6 / 11$ \\
\hline 7 & 26 & $?$ & $x$ & & $x$ & & $x$ & & $x$ & II & +++ & $31 / 33$ \\
\hline 8 & 31 & 3 & & & $x$ & & $x$ & & $x$ & 0 & +++ & $31 / 33$ \\
\hline 9 & 26 & 6 & & & $x$ & & $x$ & & $x$ & 0 & + & $6 / 11$ \\
\hline 10 & 24 & 48 & $x$ & $x$ & & & $x$ & & $x$ & III & - & - \\
\hline 11 & 27 & 24 & $x$ & & $x$ & $x$ & $x$ & & & I & ++ & - \\
\hline 12 & 27 & 3 & & & $x$ & & $x$ & & $x$ & 0 & + & - \\
\hline 13 & 29 & 2 & & & $x$ & & $x$ & & $x$ & I & + & - \\
\hline 14 & 58 & 36 & & & $x$ & & & $x$ & & 0 & ++ & 42 \\
\hline 15 & 22 & 1 & & $x$ & & $x$ & $x$ & & & III & + & - \\
\hline 16 & 24 & $?$ & $x$ & $x$ & & & $x$ & & $x$ & I & ++ & - \\
\hline 17 & 28 & 6 & & & $x$ & & $x$ & & $x$ & II & ++ & - \\
\hline 18 & 27 & $?$ & $x$ & $x$ & & & $x$ & & $x$ & III & - & $16 / 18$ \\
\hline 19 & 45 & 24 & & $x$ & & & $x$ & & $x$ & II & + & $31 / 33$ \\
\hline 20 & 24 & 2 & & $x$ & & $x$ & $x$ & & & II & ++ & $16 / 18$ \\
\hline 21 & 27 & $?$ & $x$ & $x$ & & & $x$ & & & II & + & - \\
\hline 22 & 26 & 12 & & & $x$ & & $x$ & & $x$ & III & ++ & $16 / 18$ \\
\hline 23 & 19 & ? & & $x$ & & & $x$ & & & II & ++ & $16 / 18$ \\
\hline
\end{tabular}

PIN:Penile intraepithelial neoplasia is graded as 0 if absent, and as degree I (mild), II (moderate) or III (severe).

Dermal inflammation: If present, the reaction is graded as mild $(+)$, moderate $(++)$ or severe $(+++)$.

$(67 \%)$. In our investigation we also have classified six patient biopsies exhibiting PIN without concurrent koilocytosis (35\%) as being light microscopically typical for GPVI. The validity of considering PIN I as an indicative criterion of underlying HPV infection might possibly have been deceptive if anti-inflammatory pre-treatment had not been given consequently in our patients. Yet, in two thirds (4/6; table 3) of biopsies exhibiting PIN I, koilocytosis was also detected; therefore, we do believe that a mild dysplasia in penile epidermis represents a reliable sign of HPV infection.

The fact that positive ISH signals were detected in merely $56 \%$ of the patient biopsies, and at a frequency as low as $26 \%$ in control biopsies may seem confounding, as all ISH negative biopsies had been classified as typical for GPVI by penoscopy as well as by histology evaluation. Accordingly, it seems likely that either suboptimal ISH test conditions existed, or that some other HPV type(s) than the ones being tested for potentially were pathogenetically involved. Regarding ISH

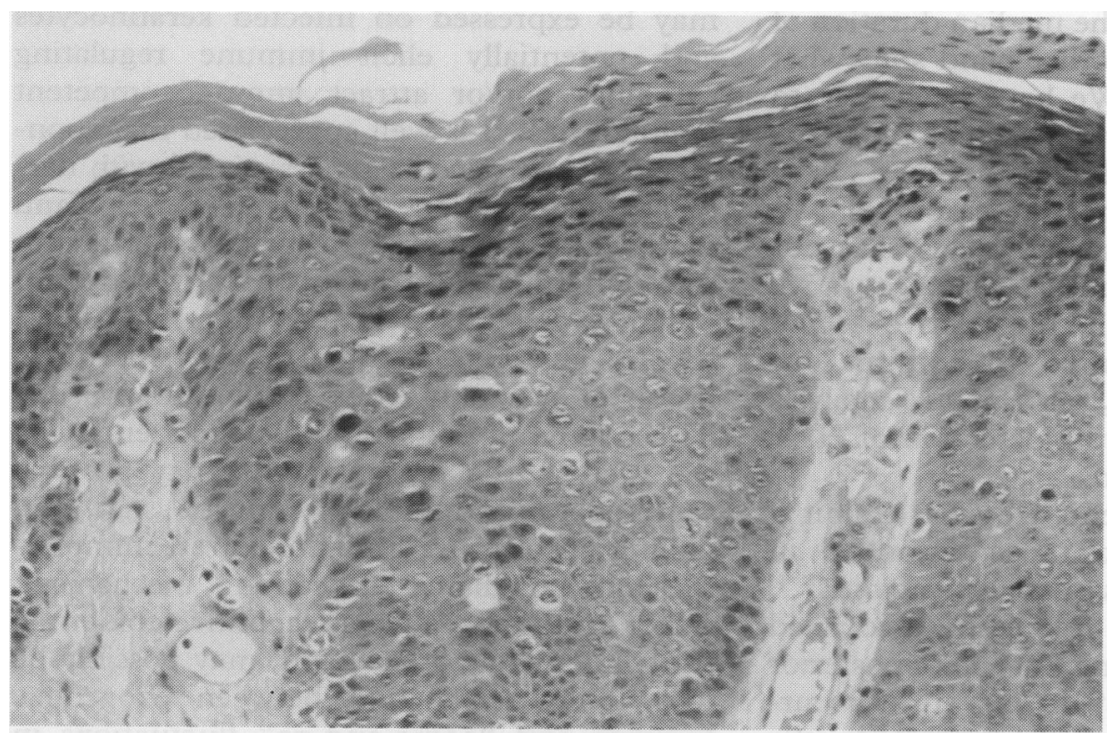

Figure 3 Histopathological picture of PIN II without any koilocytosis in this part of the epithelium. No dermal inflammatory infiltrate is present, but some dilated capillaries are seen in the upper dermal papillae. sensitivity rates, biopsy sample size is of importance, as one area of the lesion could contain HPV DNA and another not. ${ }^{25}$ The fact that we analysed relatively small samples may have been of major significance. Thus the ISH positivity rate was higher in patient samples $(4-6 \mathrm{~mm})$ than is control biopsies $(2-3$ $\mathrm{mm})$. Using $\mathrm{Ll}$ derived consensus primers, PCR positivity did not occur in any of our ISH negative patient biopsies. It is possible that the use of other types of consensus primers, ${ }^{26}$ or of nested PCR primers ${ }^{30}$ might have given different results. However, in future studies on patient categories similar to the one studied by us, we recommend a prospective design and the combined use of PCR and Southern blot assays.

The ISH positivity rate of $56 \%$ in our study falls within the lower range of $59-84 \%$ accounted for in various studies using analogous methodology applied on samples from clinically and/or histologically typical/conspicuous GPVI lesions. ${ }^{21}{ }^{21}$ However, ISH positivity also correlates with the degree of intraepithelial neoplasia. In general, a positive ISH correlation tends to exist with the presence of koilocytosis, while lower ISH positivity rates often are associated with lesions merely suggestive for HPV infection such as nuclear enlargements ${ }^{32}$ and intraepithelial neoplasia. ${ }^{31}$

In a previous ISH study by Barrasso et al,? $12 / 20(60 \%)$ of acetowhite macular lesions harboured low risk HPV types, while $8 / 20$ $(40 \%)$ were positive for oncogenic types. On the other hand, in a similar investigation ${ }^{6}$ on subclinical penile lesions we found that of 32 HPV DNA positive biopsies, as much as $17 / 32(53 \%)$ were associated with low risk types, only $7 / 32(22 \%)$ with oncogenic types, and uncharacterised HPV types in the remaining HPV DNA positive cases. The rather even distribution of benign versus oncogenic HPV types in HPV positive samples in the present series is somewhat different from our earlier findings. This difference could be due to the high number of biopsies exhibiting PIN in the present study. 
Not unexpectedly, two of the four biopsies exhibiting PIN III were all associated with the presence of oncogenic HPV types $16 / 18$ while we did not detect HPV DNA in the other two. The presence of PIN III corresponds to the "bowenoid" changes that were originally designated "bowenoid papulosis" alluding to clinically overt papular lesions in young adults of average age in the upper twenties located symmetrically on the outer genitals of both sexes. ${ }^{33-35}$ Our study underscores the view that the original description of "bowenoid papulosis" merely represents a fraction of HPV-associated severe dysplasia of the outer genital area. As accounted for also by others ${ }^{36}{ }^{37}$ these cases did not exhibit any colposcopic/penoscopic features distinguishing them from benign lesions. Interestingly, while Gross et al ${ }^{34}$ reported the presence of koilocytosis at a relatively low frequency $(5,1 \%)$ in bowenoid papulosis, we found koilocytosis in as much as 75\% (3/4; table 3 ) of lesions exhibiting PIN III.

Balanoposthitis represents a large clinical entity with a wide aetiological spectrum..$^{38}$ The most common microbiological agent is possibly various candida species, of which Candida albicans is most prevalent, but a few cases of Gardnerella vaginalis-associated balanoposthitis have also been described. ${ }^{39}$ However, in many cases the underlying cause remains unknown. While most cases of balanoposthitis represent isolated episodes of short duration, recurrent herpes simplex sometimes induces periodically recurrent balanoposthitis-like complaints. Furthermore, a number of dermatological conditions of unknown aetiology may predispose for more long-lasting symptoms; these include histologically well defined entities such as lichen sclerosus et atrophicus, lichen ruber planus and balanitis circinata parakeratotica.

The present investigation selectively includes men with a broad range of penile inflammatory symptoms including redness, itching, burning, tenderness, dyspareunia and fissuring. In the 18 men with available information the symptoms had recurrently occurred during an average of as much as 11 months (range 0-48). The median duration of symptoms was, on the other hand somewhat shorter, four months. We have been able to show some tentative evidence that might be interpreted as cause-and-effect between GPVI and such long-lasting penile symptoms. We would like to emphasise, however, that our data are still circumstantial for a causative association. Additional prospective HPV DNA studies are required to confirm our hypothesis. Preferrably, also RNA analysis should be included for the detection of HPV replication in such lesions.

The symptoms presented by us exhibit a striking resemblance to those described in females as "papillomavirus-vulvovaginitis".40 The latter condition is frequently associated with recurrent fissuring of the posterior fourchette of the vulva. ${ }^{41}$ In our study recurrent painful fissuring of the fraenulum, the coronar sulcus and/or the foreskin was reported in as much as $43 \%$ of the men, and
$39 \%$ of them had experienced periods with dyspareunia.

Accordingly, in congruence with the condition described for the vulva, we suggest the term "papillomavirus-associated balanoposthitis" for the male counterpart. The occurrence of penile oedema and inguinal adenopathy has to our knowledge previously not been associated with GPVI. It is noteworthy that $35 \%$ of these men had been treated previously and that penoscopy revealed the presence of tiny residual penile warts in $22 \%$ of them. This finding elucidates the empirical fact that current therapy often is suboptimal and that long-term problems may occur in spite of an apparent eradication of clinically visible warts.

The prevalence of HPV-associated penile symptoms is unknown. The present patient material was collected during a rather long period of time (4 years); accordingly, we feel confident that a symptomatology of the severity described here is encountered merely occasionally. Yet, inflammatory reactions associated with GPVI might indeed be more frequent than appreciated so far. ${ }^{42} 43$ Löwhagen et al ${ }^{44}$ recently reported that in a series of 88 patients with various macromorphological types of penile GPVI lesions, balanitis was found in as much as $44 \%$ of men with macular lesions (7/16). Accordingly, we would like to stress the importance of investigating the potential of an underlying GPVI in the pathogenesis of balanoposthitis, especially in persistent and/or recurrent cases as well as in men with penile lesions of unclear etiology.

In part, an inflammatory reaction may have emanated from an exogenous irritative and/or microbiological stimulus. Although PAS staining did not reveal a fungal infection in any of 15 investigated biopsies, the potential pathogenetic influence from candida cannot be ruled out due to the use of miconazol containing topical remedy prior to biopsy sampling.

The inflammatory response may also represent an HPV induced immunological response. ${ }^{45-50}$ When virus replication takes place, viral antigens and/or host neoantigens may be expressed on infected keratinocytes and potentially elicit immune regulating cytokines and/or attract immunocompetent lymphocytes. It is well appreciated that spontaneous wart rejection is associated with dermal accumulation of lymphocytes and macrophages that subsequently penetrate into the epidermis. The mere finding of dermal inflammatory cells is common in GPVI lesions $^{6}$ and may possibly represent an early phase of a spontaneous involution process. Thus, some of the symptoms experienced by the men in the present study may possibly represent a favourable prognostic sign, signalling an ongoing initiation of an immunologically mediated rejection mechanism. Other symptoms, such as repetitive epidermal fissuring, on the other hand, may possibly be due to an interaction between exogenous traumas and "come-and-go" fluctuations in virological and biological expression. ${ }^{151}$

Knowledge on the natural course of 
acetowhite lesions is quite limited. However, studies on cervical lesions indicate that the majority will clear spontaneously within a period of 5-6 years. ${ }^{10}$ For asymptomatic subclinical GPVI in men, it seems rational to await spontaneous regression of the lesions as well. However, if the lesions are associated with symptoms such as balanoposthitis, as well as for condylomas and overt bowenoid papulosis, active therapy is required and surgical eradication seems at present to represent the only alternative.

1 Schneider A. Pathogenesis of genital HPV infection. Genitourin Med 1993;69:165-73.

2 Baefverstedt B. Condylomata acuminata-past and present. Acta Dermatol Venereol 1967;47:376-81

3 Gissmann L, Wolnik L, Ikenberg H, Koldovsky U, Schnurch HG, zur Hausen H. Human papillomavirus types 6 and 11 DNA sequences in genital and laryngeal
papillomas and in some cervical cancers. Proc Natl Acad Sci USA 1983;80:560-3.

4 von Krogh G, Rylander E. Clinical Evaluation. In: von Krogh G, Rylander E, eds. GPVI. A Survey for the Krogh G, Rylander E, eds. GPVI. A Survey for the
Clinician. Karlstad, Sweden. Conpharm AB/KABI Clinician. Karlstad, Sweden. Conpharm

5 Syriänen KJ. Epidemiology of human papillomavirus (HPV) infections and their association with genital squamous cell cancer. APMIS 1989;97:957-70.

6 Wikström A, Hedblad M-A, Johansson B, et al. The acetic acid test in evaluation of subclinical genital papillomavirus infection: A comparative study on penoscopy, histopathology, virology and scanning electro microscopy findings. Genitourin Med 1992;68:90-9.

7 Barrasso R, De Brux J, Croissant O, Orth G. High prevalence of papillomavirus-associated penile intraepithelial neoplasia in sexual partners of women with cervical intraepithe

8 von Krogh G. Clinical relevance and evaluation of genitoanal papilloma virus infection in the male. Semin Dermatol 1992;11:229-40.

9 Kataja V, Syrjänen K, Mäntyjärvi R, et al. Prospective follow-up of cervical HPV infections: Life table analysis of histopathological, cytological and colposcopic data. Eur f Epidemiol 1989;5:1-7.

10 Kataja V, Syrjänen S, Mäntyjärvi R, Ylikoski M, Saarikoski $\mathrm{S}$, Syrjänen $\mathrm{K}$. Prognostic factors in cervical human papillomavirus infections. Sex Transm Dis 1992;19: pap-60.

11 Campion MJ, McCance DJ, Mitchell HS, Jenkins D, Singer A, Oriel JD. Subclinical penile human papillomavirus infection and dysplasia in consorts of women with cervical neoplasia. Genitourin Med 1988;64:90-9.

12 Schneider A, Kirchmayr R, de Villiers E-M, Gissmann L. Subclinical human papillomavirus infections in male sexual partners of female carriers. 7 Urol 1988;140: $1431-4$.

13 Schultz RE, Miller JW, MacDonald GR, et al. Clinical and molecular evaluation of acetowhite genital lesions in men. F Urol 1990;143:920-3.

14 Chow VTK, Tay SK, Tham KM, Lim-Tan SK, Bernard HU. Subclinical human papillomavirus infection of the male lower genital tract: colposcopy, histology and DNA analysis. Int $\mathcal{F}$ STD $\mathcal{E}$ AIDS 1991;2:41-5.

15 Hippeläinen M, Ylikoski M, Saarikoski S, Syrjänen K Genital human papillomavirus lesions of the male sexual partners: the diagnostic accuracy of peniscopy. Genitourin Med 1991;67:291-6.

16 Bergman A, Nalick $R$. Prevalence of human papillomavirus infection in men. Comparison of the partners of mavirus infection in men. Comparison of the partners of Medicine 1992;37:710-2.

17 Tabrizi SN, Tan J, Quinn M, Borg AJ, Garland SM. Detection of genital human papillomavirus (HPV) DNA by PCR and other conventional hybridisation techniques in male partners of women with abnormal Papanicolaou smears. Genitourin Med 1992;68:370-3.

18 Monsonego J, Zerat L, Catalan F, Coscas Y. Genital human papillomavirus infections: correlation of cytological, colposcopic and histological features with viral types in women and their male partners. Int 7 STD \& AIDS 1993;4:13-20.

19 Rock B, Shah KV, Farmer ER. A morphologic, pathologic, and virologic study of anogenital warts in men. Arch Dermatol 1992;127:495-500.

20 Lassus J, Niemi K-M, Syrjänen S, Krohn K, Ranki A. A comparison of histopathologic diagnosis and the demoncomparison of histopathologic diagnosis and the demon-
stration of Human Papillomavirus-specific DNA and stration of Human Papillomavirus-specific DNA and
proteins in penile warts. Sex Transm Dis 1992;19: proteins

21 von Krogh G, Syrjänen SM, Syrjänen KJ. Advantage of human papillomavirus typing in the clinical evaluation of genitoanal warts. F Am Acad Dermatol 1988;18:495-503.

22 Richart RM. A modified terminology for cervical intraepithelial neoplasia. Obstet Gynecol 1990;75:131-3.

23 Syriänen SM. Basic concepts and practical applications of recombinant DNA techniques in detection of human papillomavirus (HPV) infections. APMIS 1990;98: 95-110

24 Syrjänen S, Syrjänen K. An improved in situ hybridization protocol for detection of human papillomavirus (HPV) DNA sequences in paraffin-embedded sections. $\mathcal{F}$ Virol Methods 1986;14:293-304.

25 de Villiers E-M. Laboratory techniques in the investigation of human papillomavirus infection. Genitourin Med 1992;68:50-4.

26 van der Brule AJC, Snijders PJF, Meier CJLM, Walboomers JMM. PCR-based detection of genital HPV genotypes: an update and future perspectives. Papillomavirus Report 1993;4:95-9.

27 Syriänen SM, von Krogh G, Syriänen KJ. Detection of human papillomavirus DNA in anogenital condylomata in men using in situ DNA hybridisation applied to parafin men using in situ DNA hybridisation appli

28 Syrjänen S, Partanen P, Mäntyjärvi R, Syrjänen $K$. Sensitivity of in situ hybridization techniques using Sensitivity of in situ hybridization techniques using
biotin and ${ }^{35}$ S-labeled human papillomavirus (HPV) DNA probes. $\mathcal{F}$ Virol Methods 1988;19:225-38

29 Manos MM, Ting Y, Wright DK, Lewis AJ, Broker TR, Wolinsky SM. The use of polymerase chain reaction amplification for the detection of genital human papillomaviruses. Cancer Cells 1989;7:209-14.

30 Evander M. Detection of Human Papillomavirus. A study of normal cells, cervical intraepithelial neoplasia and cancer of the uterine cervix. Thesis. University of Umeå, 1991.

31 Nuovo GJ, Friedman D, Richart RM. In situ hybridization analysis of Human Papillomavirus DNA segregation patterns in lesions of the female genital tract. Gynecol Oncol terns in lesions of

32 Yang GCH, Demopoulos RI, Chan W, Mittal KR Superficial nuclear enlargement without koilocytosis as an expression of Human Papillomavirus infection of the uterine cervix: An In situ hybridization study. Int $\mathcal{F}$ Gynecol Pathol 1992;11:283-7.

33 Wade TR, Kopf AW, Ackerman AB. Bowenoid papulosis of the genitalia. Arch Dermatol 1979;115:306-8.

34 Gross G, Hagedorn M, Ikenberg H, et al. Bowenoid papulosis. Presence of human papillomavirus (HPV) structural antigens and of HPV 16-related DNA sequences. Arch Dermatol 1985;121:858-63.

35 Gimeno E, Vilata JJ, Sanchez IL, Lloret A, Fortea JM. Bowenoid papulosis: clinical and histological study of eight cases. Genitourin Med 1987;63:109-13.

36 Krebs H-B, Schneider V. Human Papillomavirus-associated lesions of the penis: Colposcopy, cytology, and histology. Obstet Gynecol 1987;70:299-304

37 Cone R, Beckmann A, Aho M, et al. Subclinical manifestations of vulvar human papillomavirus infection. Int $\mathcal{f}$ Gynecol Pathol 1991;10:26-35.

38 Hillman RJ, Walker MM, Harris JRW, Taylor-Robinson D. Penile dermatoses: a clinical and histopathological study. Genitourin Med 1992;68:166-9.

39 Burdge DR, Bowie WR, Chow AW. Gardnerella vaginalisassociated balanoposthitis. Sex Transm Dis 1986;13. 159-62.

40 Bodén E, Eriksson A, Rylander E, von Schoultz B. Clinical characteristics of papillomavirus-vulvovaginitis. Acto Obstet Gynecol Scand 1988;67:147-51.

41 Byrne MA, Walker MM, Leonard J, Pryce D, TaylorRobinson D. Recognising covert disease in women with Robinson D. Recognising covert disease in women with
chronic vulval symptoms attending an STD clinic: value chronic vulval symptoms attending an STD clinic: value of detailed examination

42 Griffiths M, Penna LK, Tovey SJ. Aceto-white change of the glans penis associated with balanitis not human papillomavirus infection. Int $\mathcal{f}$ STD $\mathcal{F}$ AIDS 1991 2:211-2.

43 Arumainayagam JT, Sumathipala AHT, Smallman LA, Shahmanesh M. Flat condyloma of the penis presenting as patchy balanoposthitis. Genitourin Med 1990;66: 251-3.

44 Löwhagen G-B, Bolmstedt A, Ryd W, Voog E. The prevalence of "high-risk" HPV types in penile condyprevalence of "high-risk" HPV types in penile condyloma-like lesions: correlation between HPV

45 Jablonska S, Majewski S, Malejczyk J. HPV infection and immunological responses. In: von Krogh G, Rylander E, eds. GPVI. Genitoanal Papillomavirus Infection. A survey for the Clinican. Karlstad, Sweden. Conpharm AB/Kabi Pharmacia AB. Chapter X, ii:289-329.

46 McMillan A, Bishop PE, Fletcher S. An immunohistological study of condylomata acuminata. Histopatho 1990;17:45-52.

47 Bishop PE, McMillan A, Fletcher S. An immunohistological study of spontaneous regression of condylomata acuminata. Genitourin Med 1990;66:79-81.

48 Tindle RW, Frazer IH. Immunology of anogenital human papillomavirus (HPV) infection. Aust NZ $\mathcal{f}$ Obstet papillomavirus (HPV)

49 Vardy DA, Baadsgaard O, Hansen ER, Lisby S, Vejlsgaard GL. The cellular immune response to human GL. The cellular immune response to human papillom

50 Roche JK, Crum CP. Local immunity and the uterine cervix: implications for cancer-associated viruses. Cancer Immunol Immunother 1991;33:203-9.

51 Schneider A, Kirchhoff T, Meinhardt G, Gissmann L. Repeated evaluation of human papillomavirus 16 status in cervical swabs of young women with a history of normal Papanicolaou smears. Obstet Gynecol 1992;79: 683-8. 